\title{
Growing Threat of Extended-Spectrum $\beta$-Lactamase-Producing Enterobacteriaceae Colonization in High-Risk Pregnancies: a retrospective cohort study
}

\author{
Yun-Sun Choi ${ }^{1}$, Yejin Kim ${ }^{1}$, Hye Jung Cho ${ }^{1}$, Ji-Hee Sung ${ }^{1}$, Suk-Joo Choi ${ }^{2}$, Soo-young Oh ${ }^{1}$, \\ Yae-Jean $\mathrm{Kim}^{1}$, and Cheong-Rae Roh ${ }^{1}$ \\ ${ }^{1}$ Samsung Medical Center, Sungkyunkwan University School of Medicine, Seoul \\ ${ }^{2}$ Samsung Medical Center, Sungkyunkwan University School of Medicine
}

November 4, 2021

\begin{abstract}
Objective To investigate the epidemiological changes in extended-spectrum $\beta$-lactamase-producing Enterobacteriaceae (ESBLE) vaginal colonization in high-risk pregnant women and identify independent risk factors. Further, the differences in perinatal outcomes according to maternal ESBL-E vaginal colonization were analyzed. Design Retrospective cohort study. Setting Republic of Korea Population 1,460 women admitted to our high-risk pregnancy unit between $14+0$ and $35+6$ gestational weeks. Methods The study period was divided into periods 1 (January 2010 to July 2015) and 2 (August 2015 to December 2020). The main outcomes were analyzed according to each period and ESBL-E vaginal colonization. Main Outcome Measures ESBL-E vaginal colonization rate, risk factors for ESBL-E vaginal colonization, and perinatal outcomes. Results The ESBL-E vaginal colonization rate was higher in period 2, which was attributed to a significantly higher proportion of ESBL-producing Escherichia coli. Cerclage (odds ratio [OR]: 3.248; 95\% confidence interval [CI]: 1.744-6.049) and prior antibiotic treatment (OR: 3.044; 95\% CI: 1.713-5.410) were found as independent risk factors for ESBL-E vaginal colonization. Earlier gestational age at delivery, and higher proven early-onset neonatal sepsis (EONS) rate were observed in the ESBL-E-positive group. Conclusions The ESBL-E vaginal colonization rate in high-risk pregnant patients has increased over the past decade, and the independent risk factors for colonization are cerclage and prior antibiotic treatment. Additionally, maternal ESBL-E vaginal colonization is associated with higher proven EONS rates. Funding This study received no funding. Keywords Extended-spectrum $\beta$-lactamase, Enterobacteriaceae, vaginal colonization, antibiotics use, cerclage, neonatal sepsis
\end{abstract}


ö

11The names are listed in order of contribution to the paper.

Yun-sun Choi, ${ }^{\mathrm{a}}$ Yejin Kim, ${ }^{\mathrm{a}}$ Hye Jung Cho, ${ }^{\mathrm{a}}$ Ji-Hee Sung, ${ }^{\mathrm{a}}$ Suk-Joo Choi, ${ }^{\mathrm{a}}$ Soo-young Oh, ${ }^{\mathrm{a}}$ Yae-Jean Kim, ${ }^{\mathrm{b}}$ Cheong-Rae Roh ${ }^{\mathrm{a}}$

${ }^{a}$ Department of Obstetrics and Gynecology, Samsung Medical Center, Sungkyunkwan University School of Medicine, Seoul, Korea

${ }^{\mathrm{b}}$ Department of Pediatrics, Samsung Medical Center, Sungkyunkwan University School of Medicine, Seoul, Korea

Running title: ESBL-E vaginal colonization in high-risk pregnancies

Correspondence to Soo-young $\mathrm{Oh}$ 
Department of Obstetrics and Gynecology, Samsung Medical Center, Sungkyunkwan University School of Medicine, Seoul, Korea

81 Irwon-ro, Gangnam-gu, Seoul 06351 Korea

E-mail: ohsymd@skku.edu.

\section{Abstract}

\section{Objective}

To investigate the epidemiological changes in extended-spectrum $\beta$-lactamase-producing Enterobacteriaceae (ESBL-E) vaginal colonization in high-risk pregnant women and identify independent risk factors. Further, the differences in perinatal outcomes according to maternal ESBL-E vaginal colonization were analyzed.

\section{Design}

Retrospective cohort study.

\section{Setting}

Republic of Korea

\section{Population}

1,460 women admitted to our high-risk pregnancy unit between $14^{+0}$ and $35^{+6}$ gestational weeks.

\section{Methods}

The study period was divided into periods 1 (January 2010 to July 2015) and 2 (August 2015 to December 2020). The main outcomes were analyzed according to each period and ESBL-E vaginal colonization.

\section{Main Outcome Measures}

ESBL-E vaginal colonization rate, risk factors for ESBL-E vaginal colonization, and perinatal outcomes.

\section{Results}

The ESBL-E vaginal colonization rate was higher in period 2, which was attributed to a significantly higher proportion of ESBL-producingEscherichia coli . Cerclage (odds ratio [OR]: 3.248; $95 \%$ confidence interval [CI]: 1.744-6.049) and prior antibiotic treatment (OR: 3.044; 95\% CI: 1.713-5.410) were found as independent risk factors for ESBL-E vaginal colonization. Earlier gestational age at delivery, and higher proven early-onset neonatal sepsis (EONS) rate were observed in the ESBL-E-positive group.

\section{Conclusions}

The ESBL-E vaginal colonization rate in high-risk pregnant patients has increased over the past decade, and the independent risk factors for colonization are cerclage and prior antibiotic treatment. Additionally, maternal ESBL-E vaginal colonization is associated with higher proven EONS rates.

\section{Funding}

This study received no funding.

\section{Keywords}

Extended-spectrum $\beta$-lactamase, Enterobacteriaceae, vaginal colonization, antibiotics use, cerclage, neonatal sepsis

\section{Introduction}

Extended-spectrum $\beta$-lactamases (ESBLs) are a heterogeneous group of enzymes produced by certain bacteria that inactivate $\beta$-lactam antibiotics via hydrolysis. They are most often found inEnterobacteriaceae, 
especially Escherichia coli and Klebsiella pneumoniae. Because of antimicrobial resistance, diagnosing and treating ESBL-producing bacterial species are important challenges. ${ }^{1,2}$

The prevalence of ESBL-producing Enterobacteriaceae (ESBL-E) infection is not exactly known; however, the reported prevalence has increased globally in recent years, ${ }^{3-5}$ while the estimated prevalence in Asia is approximately $4.8 \%$ to $12.0 \% .^{2,6-8}$ Furthermore, the rates of ESBL-E infection in maternity wards and outpatient clinics are also increasing. Therefore, these issues related to ESBL-E infection are becoming more crucial among obstetricians. ${ }^{9}$

Following the report of maternal ESBL-E vaginal colonization as a precursor of mother-to-child transmission, the importance of vaginal colonization was recently noted. ${ }^{10}$ According to a recent systematic review, neonatal ESBL-E infection in neonatal intensive care units is associated with significant morbidity and mortality, with a mortality rate as high as $31 \% .{ }^{11}$ Meanwhile, the risk factors for ESBL-E infection in the general population include prior antibiotic treatment, increased illness severity, indwelling catheter use, and intensive care unit admission. ${ }^{12,13}$ However, the specific risk factors for ESBL-E vaginal colonization during pregnancy have not been studied, and the potential clinical implications are still unclear. ${ }^{14}$

With this background, we aimed to evaluate the changes in the rate of ESBL-E vaginal colonization in highrisk pregnancies over the study period and identify the risk factors for ESBL-E vaginal colonization during pregnancy. Further, we analyzed the perinatal outcomes of ESBL-E-positive patients.

\section{Methods}

\section{Study population}

This retrospective cohort study included consecutive pregnant women who were admitted to the high-risk pregnancy unit of our institution between $14^{+0 / 7}$ and $35^{+6 / 7}$ weeks of gestation and delivered a baby at our hospital. The indications for admission were preterm premature rupture of membrane (pPROM), preterm labor (PTL), incompetent internal os of the cervix (IIOC), and short cervical length (CL) (defined as a CL of $<25 \mathrm{~mm}$ on transvaginal ultrasound). The study period was from January 2010 to December 2020 and was arbitrarily divided into period 1 (January 2010 to July 2015) and period 2 (August 2015 to December 2020 ) to evaluate changes in the association of ESBL-E vaginal colonization over the time periods. The study was approved by our institutional review board (no. 2020-08-006). The requirement for written informed consent was waived owing to the retrospective design of our study. Prior to analysis, all patient records were anonymized.

\section{Vaginal culture examinations}

Since 2010, our institution has introduced a practice protocol in which upper vaginal culture is routinely performed for high-risk patients diagnosed with pPROM, PTL, IIOC, and short CL. Vaginal culture samples were collected under sterile technique using a sterile swab and a sterile non-lubricated speculum and transported to the laboratory for analysis using a suitable transportation medium. The samples were Gramstained and cultured according to the standard laboratory procedures of our institution. They were directly inoculated on a blood agar plate, MacConkey agar plate, and Thayer-Martin agar plate and incubated at $35^{\circ} \mathrm{C}$ for up to $72 \mathrm{~h}$ in a $5 \% \mathrm{CO}_{2}$ incubator. Antimicrobial susceptibility testing, including that for ESBL-E activity, was performed using the VITEK 2 system (bioMérieux, Hazelwood, MO). Cefepime, cefotaxime, ceftazidime, cefepime/clavulanic acid, cefotaxime/clavulanic acid, and ceftazidime/clavulanic acid were included in the ESBL-E testing using the VITEK 2 system. There was no significant change in the diagnostic method for ESBL-E during the study period.

\section{Clinical characteristics of the study population}

We collected data on maternal characteristics, including age, parity, pre-pregnancy body mass index, history of preterm birth (PTB), plurality, infertility treatment (intrauterine insemination and in vitro fertilization $[\mathrm{IVF}]$ ) of index pregnancy, gestational age at admission, diagnosis at admission (pPROM, IIOC, PTL, or short CL), and use of antibiotics before admission. Prior antibiotic treatment was defined as the use of 
antibiotics within 4 weeks prior to the vaginal culture test. Perinatal outcomes included gestational age at delivery, mode of delivery, abortion, stillbirth, histologic chorioamnionitis, sex, birth weight, Apgar score, neonatal mortality, neonatal intensive care unit admission rate, and neonatal sepsis. Abortion included all fetal loss in utero occurring before 20 weeks of gestation, while stillbirth included fetal loss occurring beyond 20 weeks of gestation with 5-min Apgar scores of 0. According to the protocol used by Redline et al., ${ }^{15}$ histologic chorioamnionitis was reviewed by a single pathologist (JSK) as previously defined. ${ }^{16}$ Early-onset neonatal sepsis (EONS) was defined as neonatal sepsis within the first 3 days of life, including infection by any kind of organism. Suspected sepsis was diagnosed clinically by pediatricians, while proven sepsis was diagnosed when microorganisms were isolated from the blood or cerebrospinal fluid of neonates.

\section{Statistical analysis}

Statistical analysis was performed using Pearson's chi-square test and Fisher's exact test for categorical data and the Wilcoxon rank sum test for continuous data. Neonatal outcomes were analyzed using the generalized estimating equation method, considering twins and triplets born to the same mother. A multiple logistic regression analysis was performed to identify the independent risk factors for ESBL-E vaginal colonization. The adjustment factors were selected backward for variables with $P$-values of $<0.05$ in the univariate analysis, and a multivariate analysis was performed after confirmation that these adjustment factors had no collinearity in the covariate analysis. Statistical significance was set at $P$-values of $<0.05$. Statistical analysis was performed using SAS version 9.4 (SAS Institute, Cary, NC) and R version 4.0.4 (Vienna, Austria; http://www.R-project.org/).

\section{Results}

\section{Clinical characteristics of the study population according to the study period}

During the study period, a total of 1,460 women were eligible for this study. Table 1 summarizes the comparison of maternal ESBL-E vaginal colonization according to the study period. The overall maternal ESBL-E vaginal colonization rate was significantly higher in period 2 than in period 1 (6.2\% vs $2.4 \%, P<0.001)$. Notably, the positivity rate for E. coli colonization $(14.3 \%$ vs $6.3 \%, P<0.001)$ and the rate of ESBL-Epositive $E$. coli colonization were significantly higher in period 2 than in period $1(5.3 \%$ vs $1.7 \%, P<0.001)$. However, there was no significant difference in the rates of $K$. pneumoniae colonization and ESBL-E-positive K. pneumoniae colonization between periods 1 and 2 .

Table 2 compares the maternal baseline characteristics according to the study period. The maternal age, primiparity, pre-pregnancy body mass index, IVF-embryo transfer pregnancy rate, and short CL rate were significantly higher in period 2 than in period 1. In particular, the prior antibiotic treatment rate within 4 weeks was significantly higher in period 2 than in period $1(18.5 \%$ vs $13.9 \%, P=0.018)$. We additionally checked the indications for antibiotic use among the women with prior antibiotic treatment $(n=238)$. Suspected diagnosis or confirmed diagnosis of pPROM $(n=160)$ was most often indicated, followed by PTL, prophylactic antibiotic use before or after cerclage, IIOC, and fever, as shown in Table 2. No significant difference was observed for any indication between the two periods.

\section{Clinical characteristics of the study population according to maternal ESBL-E vaginal coloni- zation}

We also compared the maternal baseline characteristics according to ESBL-E vaginal colonization (Table 3). During the study period, 64 patients $(4.4 \%)$ were confirmed to be positive for ESBL-E based on the vaginal culture findings. As shown in Table 3, the rates of IVF-embryo transfer pregnancy, IIOC, and cerclage before admission were significantly higher in the ESBL-E-positive group. In addition, the rate of antibiotic treatment before vaginal culture was significantly higher in the ESBL-E-positive group than in the ESBLE-negative group ( $43.8 \%$ vs $15.0 \%, P<0.001)$. The gestational age at admission was significantly lower in the ESBL-E-positive group than in the ESBL-E-negative group. We compared the duration of antibiotic use in 238 patients who used antibiotics before vaginal culture according to ESBL-E vaginal colonization. The median duration of antibiotic treatment was significantly longer in the ESBL-E-positive group than in the 
ESBL-E-negative group ( $7.0 \%$ vs $4.0 \%, P=0.015)$.

\section{Independent risk factors for maternal ESBL-E vaginal colonization}

We identified the independent risk factors for the increase in ESBL-E vaginal colonization through a multivariate analysis (Table 4). The analysis showed that cerclage (odds ratio [95\% confidence interval]: 3.248 [1.744-6.049]) and prior antibiotic treatment (odds ratio [95\% confidence interval]: 3.044 [1.713-5.410]) remained as significant risk factors for positive ESBL-E vaginal colonization.

\section{Perinatal outcomes according to maternal ESBL-E vaginal colonization}

Finally, we compared the perinatal outcomes according to ESBL-E vaginal colonization in 1,460 mothers and 1,616 live-born neonates. As shown in Table 5, the gestational age at admission and delivery was significantly earlier, and the rates of abortion and histologic chorioamnionitis were significantly higher in the ESBL-Epositive group than in the ESBL-E-negative group. The overall rate of EONS $(7.6 \%$ vs $2.5 \%, P=0.040)$ and the rate of proven EONS were significantly higher in the ESBL-E-positive group than in the ESBL-E-negative group $(6.0 \%$ vs $1.1 \%, P=0.002)$.

\section{Discussion}

\section{Main Findings}

Our study clearly showed a significant increase in the rate of ESBL-E vaginal colonization, especially ESBLproducing $E$. coli , in high-risk pregnancies over the past decade. We also demonstrated that prior antibiotic treatment and cerclage were the independent risk factors for ESBL-E vaginal colonization after adjusting for confounding variables. In addition, maternal ESBL-E vaginal colonization was associated with earlier gestational age at admission and delivery and higher rate of EONS, especially in cases of proven sepsis.

\section{Strengths and Limitations}

Our study has some limitations. First, since we analyzed the relationship between the results of vaginal culture at admission and neonatal outcomes, our analysis did not consider antibiotic treatment during hospitalization, which may affect the neonatal outcomes. Second, although cerclage was identified as a risk factor for ESBL-E vaginal colonization, the causality is uncertain. In fact, a previous study has demonstrated that the proportion of Lactobacillus spp. was reduced, while that of abnormal vaginal flora increased before rescue cerclage. ${ }^{28}$ Considering this result, the possibility that ESBL-E vaginal colonization had already occurred before the operation could not be excluded because information on vaginal culture before cerclage was not available in this study population.

To the best of our knowledge, this is the first study to identify changes in the prevalence and risk factors of ESBL-E vaginal colonization in high-risk pregnancies. Our study is also meaningful in that we included a relatively large number of patients who underwent vaginal culture according to the protocol of our institution. In addition, the need for further studies on the treatment of ESBL-E colonization and pregnancy outcomes was identified in this study. For example, there are only a few randomized controlled trials on ESBL-E infection treatment, and there are many controversies on whether antibiotic treatment for ESBL-E infection is effective or on which treatment methods are effective. ${ }^{45,46}$

\section{Interpretation}

New $\beta$-lactamases have emerged rapidly with the development of new $\beta$-lactam antibiotics over the past 20 years, and the prevalence of ESBL-producing bacteria is increasing worldwide. ${ }^{1,3-5}$ The prevalence of community-acquired ESBL-E infections among hospitalized patients has also increased in Korea. ${ }^{17}$ In our study, the rate of maternal ESBL-E vaginal colonization increased to $6.2 \%$ in the recent period. In a Norwegian study, the prevalence of ESBL-E rectal colonization was $2.9 \%$ among pregnant women at 36 weeks of gestation. ${ }^{18}$ In another study conducted in Sri Lanka, the pre-delivery ESBL-E lower vaginal colonization rate was $1.6 \% .{ }^{19}$ 
Our data are in line with previously reported findings that prior use of antibiotics, particularly fluoroquinolone and cephalosporin, is a risk factor for ESBL-E infection in hospitalized patients. ${ }^{1,2}$ It is well recognized that an increase in the rate of ESBL-E infection is associated with the widespread use of antibiotics. ${ }^{1,2,20-22}$ In our study, the multivariate analysis confirmed that prior antibiotic treatment and cerclage were the independent risk factors for ESBL-E vaginal colonization in high-risk pregnant patients. In fact, previous studies have suggested that antibiotic administration during pregnancy may alter the vaginal microbiological ecology. ${ }^{23,24}$ For example, lessLactobacillus species colonization than expected was reported in pregnant women using antibiotics, and an increased vaginal colonization rate by $E$. coli was reported after antibiotic use during pregnancy. ${ }^{24}$ Similar to the general population, prolonged antibiotic use in pregnant women is also a known risk factor for ESBL-producing bacterial infection. ${ }^{12,13}$ Similarly, our study also showed that the duration of antibiotic administration was significantly longer in the ESBL-E-positive group. Clinically, the proper use of antibiotics during pregnancy is very important for the health of both the mother and fetus. ${ }^{25}$ Considering our study results, antibiotic treatment during pregnancy could be a risk factor for ESBL-E vaginal colonization infection and thus should be used only when needed. Therefore, our study also indirectly supports the limited exposure to antibiotics during pregnancy with narrow-spectrum antibiotics, if possible.

Cerclage is an effective treatment option for preventing PTB in women with cervical incompetency. ${ }^{26,27}$ In several studies, dilated cervix and short CL were associated with a paucity ofLactobacillus species in the vagina. ${ }^{28,29}$ A reduced abundance of normal vaginal flora could make it vulnerable to colonization by abnormal vaginal flora. Previous studies have suggested that cerclage increases the risk of maternal infection requiring medical intervention and puerperal pyrexia ${ }^{27,30}$; the possibility of vaginal dysbiosis owing to the effects of suture materials has also been suggested. ${ }^{31}$ Our study found that cerclage was associated with the risk of ESBL-E vaginal colonization. However, it is unclear whether cerclage is the cause or result of ESBL-E vaginal colonization. Similarly, one study confirmed that when postoperative vaginal culture was performed in patients undergoing cerclage, more than $75 \%$ of the samples were positive for $E$. coli. ${ }^{32}$ Based on the results of these studies, it is necessary to consider the risks and benefits when deciding whether to perform cerclage.

Abnormal vaginal colonization by $E$. coli or $K$. pneumoniaeduring pregnancy is clinically important with respect to neonatal infection and PTB. ${ }^{33-36}$ Especially, ESBL-E is very important because it has been reported to be the major cause of neonatal infection, which increases neonatal mortality owing to limitations in therapeutic options. ${ }^{10,37,38}$ In light of this importance, it is considerably regrettable that there are only a few studies on the screening or treatment of ESBL-E colonization in pregnant women.

The organisms known to cause EONS are generally colonized in the maternal genito-urinary tract. ${ }^{39}$ Among them, maternal $E$. coli vaginal colonization has been widely studied as an independent risk factor for neonatal E. colisepsis. ${ }^{40,41}$ In addition, a previous study has shown that EONS with $E$. coli was associated with worse perinatal outcomes compared with EONS with group BStreptococcus. ${ }^{42}$ For this reason, neonatalE. coli sepsis, especially ESBL-E sepsis, which is difficult to treat, is emerging as an important condition. A recent study on ESBL-E infection during pregnancy identified that maternal ESBL-E colonization is an important risk factor for vertical transmission. The authors explained that the rate of ESBL-E-positive postnatal swabs was significantly higher in neonates born to mothers who were positive for ESBL-E colonization at delivery. ${ }^{43}$ Among the total neonatal sepsis cases in the ESBL-E-positive group in our study, ESBL-E was identified in five cases. Consequently, the mother-to-neonate transmission rate of ESBL-E infection was estimated to be $7.6 \%(5 / 66)$. According to a Sri Lankan study, the mother-to-neonate transmission rate of Enterobacteriaceae was $6.9 \%$, of which ESBL producers had a transmission rate of $0.6 \%{ }^{19}$ Another study reported a mother-to-neonate ESBL-E transmission rate of $34.3 \% .{ }^{44}$ Our study also confirmed that the rate of proven EONS was significantly higher in the ESBL-E-colonized mothers. This finding supports the results of previous studies and highlights the importance of evaluating the need for screening and treatment for ESBL-E colonization in high-risk pregnancies.

\section{CONCLUSION}


To date, upper vaginal culture is not routinely recommended even in high-risk pregnancies ${ }^{47,48}$; nevertheless, screening for high-risk pregnancies may be justified if there is evidence that treatment of ESBL-E vaginal colonization improves neonatal outcomes. Therefore, we suggest the need for further studies on screening and treatment of ESBL-E vaginal colonization to improve the perinatal outcomes against the increasing threat of ESBL-E colonization during pregnancy. In conclusion, our findings indicate that judicious use of antibiotics and prudent cerclage are needed.

\section{DISCLOSURE OF INTEREST}

The authors have no conflict of interest to disclose.

\section{CONTRIBUTION TO AUTHORSHIP}

YC wrote the manuscript and researched data. YK researched data. HC researched data. JS researched data and reviewed/edited the manuscript. SC reviewed/edited the manuscript. SO contributed to the discussion and reviewed/edited the manuscript. YK reviewed/edited the manuscript. CR reviewed/edited the manuscript.

\section{ETHICS APPROVAL}

The study protocol was approved by our institutional review board (no. 2020-08-006) and followed the principles of Declaration of Helsinki. The requirement for written informed consent was waived owing to the retrospective design of our study.

\section{References}

1 Bradford PA. Extended-spectrum beta-lactamases in the 21st century: characterization, epidemiology, and detection of this important resistance threat. Clin Microbiol Rev 2001;14:933-51, table of contents.

2 Ghafourian S, Sadeghifard N, Soheili S, Sekawi Z. Extended Spectrum Beta-lactamases: Definition, Classification and Epidemiology. Curr Issues Mol Biol 2015;17:11-21.

3 Pitout JD, Laupland KB. Extended-spectrum beta-lactamase-producing Enterobacteriaceae: an emerging public-health concern. Lancet Infect Dis 2008;8:159-66.

4 Canton R, Novais A, Valverde A, Machado E, Peixe L, Baquero F, et al. Prevalence and spread of extended-spectrum beta-lactamase-producing Enterobacteriaceae in Europe. Clin Microbiol Infect 2008;14 Suppl 1:144-53.

5 Woerther PL, Burdet C, Chachaty E, Andremont A. Trends in human fecal carriage of extended-spectrum beta-lactamases in the community: toward the globalization of CTX-M. Clin Microbiol Rev2013;26:744-58.

6 Pai H, Lyu S, Lee JH, Kim J, Kwon Y, Kim JW, et al. Survey of extended-spectrum beta-lactamases in clinical isolates of Escherichia coli and Klebsiella pneumoniae: prevalence of TEM-52 in Korea. J Clin Microbiol 1999;37:1758-63.

7 Yan JJ, Wu SM, Tsai SH, Wu JJ, Su IJ. Prevalence of SHV-12 among clinical isolates of Klebsiella pneumoniae producing extended-spectrum beta-lactamases and identification of a novel AmpC enzyme (CMY-8) in Southern Taiwan. Antimicrob Agents Chemother2000;44:1438-42.

8 Ho PL, Tsang DN, Que TL, Ho M, Yuen KY. Comparison of screening methods for detection of extendedspectrum beta-lactamases and their prevalence among Escherichia coli and Klebsiella species in Hong Kong. APMIS 2000;108:237-40.

9 Eppes CS, Clark SL. Extended-spectrum beta-lactamase infections during pregnancy: a growing threat. Am J Obstet Gynecol 2015;213:650-2.

10 Denkel LA, Schwab F, Kola A, Leistner R, Garten L, von Weizsacker K, et al. The mother as most important risk factor for colonization of very low birth weight (VLBW) infants with extended-spectrum beta-lactamase-producing Enterobacteriaceae (ESBL-E).J Antimicrob Chemother 2014;69:2230-7. 
11 Stapleton PJ, Murphy M, McCallion N, Brennan M, Cunney R, Drew RJ. Outbreaks of extended spectrum beta-lactamase-producing Enterobacteriaceae in neonatal intensive care units: a systematic review. Arch Dis Child Fetal Neonatal Ed 2016;101:F72-8.

12 Rodriguez-Bano J, Picon E, Gijon P, Hernandez JR, Ruiz M, Pena C, et al. Community-onset bacteremia due to extended-spectrum beta-lactamase-producing Escherichia coli: risk factors and prognosis. Clin Infect Dis 2010;50:40-8.

13 Papst L, Beovic B, Seme K, Pirs M. Two-year prospective evaluation of colonization with extendedspectrum beta-lactamase-producing Enterobacteriaceae: time course and risk factors. Infect Dis (Lond) $2015 ; 47: 618-24$.

14 Seale J, Millar M. Perinatal vertical transmission of antibiotic-resistant bacteria: a systematic review and proposed research strategy. BJOG 2014;121:923-8.

15 Redline RW, Heller D, Keating S, Kingdom J. Placental diagnostic criteria and clinical correlation-a workshop report.Placenta 2005;26 Suppl A:S114-7.

16 Lee Y, Kim HJ, Choi SJ, Oh SY, Kim JS, Roh CR, et al. Is there a stepwise increase in neonatal morbidities according to histological stage (or grade) of acute chorioamnionitis and funisitis?: effect of gestational age at delivery. J Perinat Med2015;43:259-67.

17 Baek YJ, Kim YA, Kim D, Shin JH, Uh Y, Shin KS, et al. Risk Factors for Extended-Spectrum-betaLactamase-Producing Escherichia coli in Community-Onset Bloodstream Infection: Impact on Long-Term Care Hospitals in Korea. Ann Lab Med 2021;41:455-62.

18 Rettedal S, Lohr IH, Bernhoff E, Natas OB, Sundsfjord A, Oymar K. Extended-spectrum beta-lactamaseproducing Enterobacteriaceae among pregnant women in Norway: prevalence and maternal-neonatal transmission. J Perinatol 2015;35:907-12.

19 Nanayakkara D, Liyanapathirana V, Kandauda C, Gihan C, Ekanayake A, Adasooriya D. Maternal vaginal colonization with selected potential pathogens of neonatal sepsis in the era of antimicrobial resistance, a single center experience from Sri Lanka. BMC Infect Dis 2018;18:351.

20 Kang CI, Wi YM, Lee MY, Ko KS, Chung DR, Peck KR, et al. Epidemiology and risk factors of community onset infections caused by extended-spectrum beta-lactamase-producing Escherichia coli strains. $J$ Clin Microbiol 2012;50:312-7.

21 Kang CI, Cheong HS, Chung DR, Peck KR, Song JH, Oh MD, et al. Clinical features and outcome of community-onset bloodstream infections caused by extended-spectrum beta-lactamase-producing Escherichia coli. Eur J Clin Microbiol Infect Dis 2008;27:85-8.

22 Park SY, Kang CI, Wi YM, Chung DR, Peck KR, Lee NY, et al. Risk factors and molecular epidemiology of community-onset, multidrug resistance extended-spectrum beta-lactamase-producing Escherichia coli infections. Korean J Intern Med 2017;32:146-57.

23 Kuperman AA, Koren O. Antibiotic use during pregnancy: how bad is it? BMC Med 2016;14:91.

24 Stokholm J, Schjorring S, Eskildsen CE, Pedersen L, Bischoff AL, Folsgaard N, et al. Antibiotic use during pregnancy alters the commensal vaginal microbiota. Clin Microbiol Infect2014;20:629-35.

25 Martinez de Tejada B. Antibiotic use and misuse during pregnancy and delivery: benefits and risks. Int J Environ Res Public Health 2014;11:7993-8009.

26 Berghella V, Rafael TJ, Szychowski JM, Rust OA, Owen J. Cerclage for short cervix on ultrasonography in women with singleton gestations and previous preterm birth: a meta-analysis. Obstet Gynecol 2011;117:66371. 
27 Alfirevic Z, Stampalija T, Roberts D, Jorgensen AL. Cervical stitch (cerclage) for preventing preterm birth in singleton pregnancy. Cochrane Database Syst Rev 2012:CD008991.

28 Brown RG, Chan D, Terzidou V, Lee YS, Smith A, Marchesi JR, et al. Prospective observational study of vaginal microbiota pre- and post-rescue cervical cerclage. BJOG 2019;126:916-25.

29 Gerson KD, McCarthy C, Elovitz MA, Ravel J, Sammel MD, Burris HH. Cervicovaginal microbial communities deficient in Lactobacillus species are associated with second trimester short cervix.Am J Obstet Gynecol 2020;222:491 e1- e8.

30 Quinn M. Final report of the MRC/RCOG randomised controlled trial of cervical cerclage. $\mathrm{Br} J$ Obstet Gynaecol 1993;100:1154-5.

31 Kindinger LM, MacIntyre DA, Lee YS, Marchesi JR, Smith A, McDonald JA, et al. Relationship between vaginal microbial dysbiosis, inflammation, and pregnancy outcomes in cervical cerclage. Sci Transl Med 2016;8:350ra102.

32 Kessler I, Shoham Z, Lancet M, Blickstein I, Yemini M, Miskin A, et al. Complications associated with genital colonization in pregnancies with and without cerclage. Int J Gynaecol Obstet1988;27:359-63.

33 Carey JC, Klebanoff MA. Is a change in the vaginal flora associated with an increased risk of preterm birth? Am J Obstet Gynecol 2005;192:1341-6; discussion 1346-7.

34 Donders GGG, Bellen G, Grinceviciene S, Ruban K, Vieira-Baptista P. Aerobic vaginitis: no longer a stranger. Res Microbiol 2017;168:845-58.

35 Krohn MA, Thwin SS, Rabe LK, Brown Z, Hillier SL. Vaginal colonization by Escherichia coli as a risk factor for very low birth weight delivery and other perinatal complications. J Infect Dis 1997;175:606-10.

36 Saez-Lopez E, Guiral E, Fernandez-Orth D, Villanueva S, Gonce A, Lopez M, et al. Vaginal versus Obstetric Infection Escherichia coli Isolates among Pregnant Women: Antimicrobial Resistance and Genetic Virulence Profile. PLoS One 2016;11:e0146531.

37 Hyle EP, Lipworth AD, Zaoutis TE, Nachamkin I, Fishman NO, Bilker WB, et al. Risk factors for increasing multidrug resistance among extended-spectrum beta-lactamase-producing Escherichia coli and Klebsiella species. Clin Infect Dis 2005;40:1317-24.

38 Koizumi A, Maruyama K, Ohki Y, Nakayama A, Yamada Y, Kurosawa H, et al. Prevalence and Risk Factor for Antibiotic-resistant Escherichia coli Colonization at Birth in Premature Infants: A Prospective Cohort Study. Pediatr Infect Dis J 2020;39:546-52.

39 Cools P. The role of Escherichia coli in reproductive health: state of the art. Res Microbiol 2017;168:892901.

40 Kim JY, Sung JH, Chang KH, Choi SJ, Oh SY, Roh CR, et al. Abnormal vaginal colonization by gramnegative bacteria is significantly higher in pregnancy conceived through infertility treatment compared to natural pregnancy. J Matern Fetal Neonatal Med 2017;30:556-61.

41 Tameliene R, Barcaite E, Stoniene D, Buinauskiene J, Markuniene E, Kudreviciene A, et al. Escherichia coli colonization in neonates: prevalence, perinatal transmission, antimicrobial susceptibility, and risk factors. Medicina (Kaunas) 2012;48:71-6.

42 Mayor-Lynn K, Gonzalez-Quintero VH, O'Sullivan MJ, Hartstein AI, Roger S, Tamayo M. Comparison of early-onset neonatal sepsis caused by Escherichia coli and group B Streptococcus. Am J Obstet Gynecol 2005;192:1437-9.

43 Foessleitner P, Gasser J, Kiss H, Flunt A, Presterl E, Petricevic L, et al. Vaginal colonization of extendedspectrum beta-lactamase-producing bacteria during pregnancy: An observational study. Eur J Obstet Gynecol Reprod Biol 2020;246:86-9. 
44 Danino D, Melamed R, Sterer B, Porat N, Hazan G, Gushanski A, et al. Mother-to-child transmission of extended-spectrum-beta-lactamase-producing Enterobacteriaceae. J Hosp Infect 2018;100:40-6.

45 Seo YB, Lee J, Kim YK, Lee SS, Lee JA, Kim HY, et al. Randomized controlled trial of piperacillintazobactam, cefepime and ertapenem for the treatment of urinary tract infection caused by extendedspectrum beta-lactamase-producing Escherichia coli. BMC Infect Dis 2017;17:404.

46 Ljungquist O, Kampmann C, Resman F, Riesbeck K, Tham J. Probiotics for intestinal decolonization of ESBL-producing Enterobacteriaceae: a randomized, placebo-controlled clinical trial. Clin Microbiol Infect 2020;26:456-62.

47 American College of O, Gynecologists' Committee on Practice B-O. Practice Bulletin No. 171: Management of Preterm Labor. Obstet Gynecol 2016;128:e155-64.

48 Nygren P, Fu R, Freeman M, Bougatsos C, Klebanoff M, Guise JM, et al. Evidence on the benefits and harms of screening and treating pregnant women who are asymptomatic for bacterial vaginosis: an update review for the U.S. Preventive Services Task Force. Ann Intern Med 2008;148:220-33.

Table 1. Comparison of maternal ESBL-E vaginal colonization according to the study period

\begin{tabular}{llll}
\hline & $\begin{array}{l}\text { Period 1 January 2010 } \\
\text { to July 2015 }(\boldsymbol{n}=\end{array}$ & $\begin{array}{l}\text { Period 2 August 2015 } \\
\text { to July 2020 }(\boldsymbol{n}=\end{array}$ & P-value \\
\hline $\begin{array}{l}\mathbf{7 5 6}) \\
\text { Total }(\boldsymbol{n}=\mathbf{1 , 4 6 0})\end{array}$ & $\mathbf{7 0 4})$ & $6.2(47 / 756)$ & $<0.001^{*}$ \\
\hline $\begin{array}{l}\text { Positive for ESBL-E } \\
\text { Escherichia coli }(\%)\end{array}$ & $2.4(17 / 704)$ & $14.3(108 / 756)$ & $<0.001^{*}$ \\
$\begin{array}{l}\text { ESBL-positive } \\
\text { Escherichia coli }(\%)\end{array}$ & $1.7(12 / 704)$ & $5.3(40 / 756)$ & $<0.001^{*}$ \\
$\begin{array}{l}\text { Klebsiella pneumonia } \\
(\%)\end{array}$ & $2.3(16 / 704)$ & $3.3(25 / 756)$ & $0.232^{*}$ \\
$\begin{array}{l}\text { ESBL-positive Klebsiella } \\
\text { pneumonia }(\%)\end{array}$ & $0.7(5 / 704)$ & $1.1(8 / 756)$ & $0.479^{*}$ \\
\hline
\end{tabular}

*Pearson's chi-square test.

Data are presented as numbers (percentages).

ESBL-E, extended-spectrum $\beta$-lactamase-producing

Enterobacteriaceae

Table 2. Maternal baseline characteristics according to the study period

\begin{tabular}{|c|c|c|c|}
\hline Total $(n=1,376)$ & $\begin{array}{l}\text { Period } 1 \text { January } 2010 \\
\text { to July } 2015(n= \\
704)\end{array}$ & $\begin{array}{l}\text { Period } 2 \text { August } 2015 \\
\text { to July } 2020(n= \\
756)\end{array}$ & $P$-value \\
\hline Maternal age (yr) & $32(30-35)$ & $33(31-36)$ & $<0.001^{*}$ \\
\hline Primiparity $(\%)$ & $41.5(292 / 704)$ & $51.2(387 / 756)$ & $<0.001^{* *}$ \\
\hline $\begin{array}{l}\text { Body mass index } \\
\left(\mathrm{kg} / \mathrm{m}^{2}\right) \text { before } \\
\text { pregnancy }\end{array}$ & $20.7(19.3-22.8)$ & $21.3(19.5-24.2)$ & $<0.001^{*}$ \\
\hline $\begin{array}{l}\text { History of preterm } \\
\text { delivery (\%) } \\
\text { Multifetal pregnancy }\end{array}$ & $11.5(81 / 704)$ & $9.39(71 / 756)$ & $0.186^{* *}$ \\
\hline
\end{tabular}




\begin{tabular}{|c|c|c|c|}
\hline Total $(n=1,376)$ & $\begin{array}{l}\text { Period 1 January } 2010 \\
\text { to July } 2015(n= \\
704)\end{array}$ & $\begin{array}{l}\text { Period } 2 \text { August } 2015 \\
\text { to July } 2020(n= \\
756)\end{array}$ & $P$-value \\
\hline Twin $(\%)$ & $18.6(131 / 704)$ & $18.7(141 / 756)$ & $0.983^{* *}$ \\
\hline Triplet (\%) & $1.4(10 / 704)$ & $2.1(16 / 756)$ & $0.315^{* *}$ \\
\hline $\begin{array}{l}\text { Pregnancy by in vitro } \\
\text { fertilization-embryo } \\
\text { transfer }(\%)\end{array}$ & $15.8(111 / 704)$ & $20.2(153 / 756)$ & $0.027^{* *}$ \\
\hline $\begin{array}{l}\text { Pregnancy by } \\
\text { intrauterine } \\
\text { insemination }(\%)\end{array}$ & $2.3(16 / 704)$ & $2.3(17 / 756)$ & $0.972^{* *}$ \\
\hline $\begin{array}{l}\text { Gestational age at } \\
\text { admission (wk) } \\
\text { Diagnosis at admission }\end{array}$ & $28.2(24.6-32.4)$ & $29.3(24.1-33.1)$ & $0.097^{*}$ \\
\hline $\begin{array}{l}\text { Preterm premature } \\
\text { rupture of membrane }(\%)\end{array}$ & $39.6(279 / 704)$ & $40.0(302 / 756)$ & $0.902^{* *}$ \\
\hline $\begin{array}{l}\text { Incompetent internal os } \\
\text { of the cervix }(\%)\end{array}$ & $16.6(117 / 704)$ & $18.7(141 / 756)$ & $0.309^{* *}$ \\
\hline Cerclage (\%) & $9.0(63 / 704)$ & $11.2(85 / 756)$ & $0.147^{* *}$ \\
\hline Preterm labor (\%) & $41.3(291 / 704)$ & $35.7(270 / 756)$ & $0.027^{* *}$ \\
\hline $\begin{array}{l}\text { Short cervical length } \\
(\%)\end{array}$ & $2.6(18 / 704)$ & $8.5(64 / 756)$ & $<0.001^{* *}$ \\
\hline $\begin{array}{l}\text { Prior antibiotic } \\
\text { treatment }(\%)\end{array}$ & $13.9(98 / 704)$ & $18.5(140 / 756)$ & $0.018^{* *}$ \\
\hline
\end{tabular}

*Wilcoxon rank-sum test, ${ }^{* *}$ Pearson's chi-square test.

Data are presented as nos. (percentages) and medians (interquartile ranges).

Table 3. Maternal baseline characteristics according to maternal ESBL-E vaginal colonization

\begin{tabular}{|c|c|c|c|c|}
\hline Total $(n=1,460)$ & $\begin{array}{l}\text { Negative for } \\
\text { ESBL-E }(n= \\
1,396)\end{array}$ & $\begin{array}{l}\text { Negative for } \\
\text { ESBL-E }(n= \\
1,396)\end{array}$ & $\begin{array}{l}\text { Positive for } \\
\text { ESBL-E }(n=64)\end{array}$ & $P$-value \\
\hline Maternal age (yr) & $33(30-36)$ & $33(30-36)$ & $34(32-36)$ & $0.078^{*}$ \\
\hline Primiparity $(\%)$ & $46.7(652 / 1,396)$ & $46.7(652 / 1,396)$ & $42.2(27 / 64)$ & $0.479^{* *}$ \\
\hline $\begin{array}{l}\text { Body mass index } \\
\left(\mathrm{kg} / \mathrm{m}^{2}\right) \text { before } \\
\text { pregnancy }\end{array}$ & $21.0(19.4-23.4)$ & $21.0(19.4-23.4)$ & $21.5(19.1-25.9)$ & $0.425^{*}$ \\
\hline $\begin{array}{l}\text { History of } \\
\text { preterm delivery } \\
(\%) \\
\text { Multifetal } \\
\text { pregnancy }\end{array}$ & $10.0(140 / 1,396)$ & $10.0(140 / 1,396)$ & $18.8(12 / 64)$ & $0.026^{* *}$ \\
\hline Twin $(\%)$ & $18.5(258 / 1,396)$ & $18.5(258 / 1,396)$ & $21.9(14 / 64)$ & $0.495^{* *}$ \\
\hline Triplet (\%) & $1.7(24 / 1,396)$ & $3.1(2 / 64)$ & $3.1(2 / 64)$ & $0.406^{* * *}$ \\
\hline $\begin{array}{l}\text { Pregnancy by in } \\
\text { vitro fertilization- } \\
\text { embryo transfer } \\
(\%)\end{array}$ & $17.6(245 / 1,396)$ & $29.7(19 / 64)$ & $29.7(19 / 64)$ & $0.014^{* *}$ \\
\hline
\end{tabular}




\begin{tabular}{|c|c|c|c|c|}
\hline Total $(n=1,460)$ & $\begin{array}{l}\text { Negative for } \\
\text { ESBL-E }(n= \\
1,396)\end{array}$ & $\begin{array}{l}\text { Negative for } \\
\text { ESBL-E }(n= \\
1,396)\end{array}$ & $\begin{array}{l}\text { Positive for } \\
\text { ESBL-E }(n=64)\end{array}$ & $P$-value \\
\hline $\begin{array}{l}\text { Pregnancy by } \\
\text { intrauterine } \\
\text { insemination }(\%)\end{array}$ & $2.2(31 / 1,396)$ & $3.1(2 / 64)$ & $3.1(2 / 64)$ & $0.634^{* * *}$ \\
\hline $\begin{array}{l}\text { Gestational age at } \\
\text { admission (wk) } \\
\text { Diagnosis at } \\
\text { admission }\end{array}$ & $29.00(24.6-32.9)$ & $24.3(20.9-26.9)$ & $24.3(20.9-26.9)$ & $<0.001^{*}$ \\
\hline $\begin{array}{l}\text { Preterm premature } \\
\text { rupture of } \\
\text { membrane }(\%)\end{array}$ & $39.8(556 / 1,396)$ & $39.1(25 / 64)$ & $39.1(25 / 64)$ & $0.903^{* *}$ \\
\hline $\begin{array}{l}\text { Incompetent } \\
\text { internal os of the } \\
\text { cervix }(\%)\end{array}$ & $16.8(235 / 1,396)$ & $35.94(23 / 64)$ & $35.94(23 / 64)$ & $<0.001^{* *}$ \\
\hline Cerclage (\%) & $9.2(129 / 1,396)$ & $29.7(19 / 64)$ & $29.7(19 / 64)$ & $<0.001^{* *}$ \\
\hline $\begin{array}{l}\text { Preterm labor } \\
(\%)\end{array}$ & $38.9(543 / 1,396)$ & $28.1(18 / 64)$ & $28.1(18 / 64)$ & $0.083^{* *}$ \\
\hline $\begin{array}{l}\text { Short cervical } \\
\text { length }(\%)\end{array}$ & $5.6(78 / 1,396)$ & $6.3(4 / 64)$ & $6.3(4 / 64)$ & $0.822^{* * *}$ \\
\hline $\begin{array}{l}\text { Prior antibiotic } \\
\text { treatment }(\%)\end{array}$ & $15.0(210 / 1,396)$ & $43.8(28 / 64)$ & $43.8(28 / 64)$ & $<0.001^{* *}$ \\
\hline $\begin{array}{l}\text { Duration of prior } \\
\text { antibiotic } \\
\text { treatment (d) }\end{array}$ & $4.0(2.0-8.0)$ & $7.0(5.0-12.0)$ & $7.0(5.0-12.0)$ & $0.015^{*}$ \\
\hline
\end{tabular}

*Wilcoxon rank-sum test, **Pearson's chi-square test, ***Fisher's exact test.

Data are presented as nos. (percentages) and medians (interquartile ranges).

ESBL-E, extended-spectrum $\beta$-lactamase-producing

Enterobacteriaceae.

\begin{tabular}{|c|c|c|}
\hline Variables &  & $P$-value \\
\hline Preg & $1.679(0.917-3.076)$ & 0.104 \\
\hline Cerclage before vaginal culture & $3.248(1.744-6.049)$ & $<0.001$ \\
\hline Prior antibiotic treatment & $3.044(1.713-5.410)$ & $<0.001$ \\
\hline
\end{tabular}

Table 4. Multivariate logistic regression analysis of the risk factors for extended-spectrum $\beta$-lactamaseproducing Enterobacteriaceae colonization

Table 5. Comparison of the perinatal outcomes according to maternal ESBL-E vaginal colonization

\begin{tabular}{llll}
\hline Total $(\boldsymbol{n}=\mathbf{1 , 4 6 0})$ & $\begin{array}{l}\text { Negative for ESBL-E } \\
(\boldsymbol{n}=\mathbf{1 , 3 9 6})\end{array}$ & $\begin{array}{l}\text { Positive for ESBL-E } \\
(\boldsymbol{n}=\mathbf{6 4})\end{array}$ & $P$-value \\
\hline $\begin{array}{l}\text { Gestational age at } \\
\text { delivery (wk) }\end{array}$ & $31.0(26.1-33.9)$ & $26.6(23.0-31.8)$ & $<0.001^{*}$ \\
$\begin{array}{l}\text { Cesarean section (\%) } \\
51.9(724 / 1,396)\end{array}$ & $56.3(36 / 64)$ & $0.492^{* *}$
\end{tabular}




\begin{tabular}{|c|c|c|c|}
\hline Total $(n=1,460)$ & $\begin{array}{l}\text { Negative for ESBL-E } \\
(n=1,396)\end{array}$ & $\begin{array}{l}\text { Positive for ESBL-E } \\
(n=64)\end{array}$ & $P$-value \\
\hline Abortion (\%) & $4.0(56 / 1,396)$ & $9.38(6 / 64)$ & $0.038^{* *}$ \\
\hline Stillbirth (\%) & $4.4(62 / 1,396)$ & $7.8(5 / 64)$ & $0.208^{* *}$ \\
\hline $\begin{array}{l}\text { Histologic } \\
\text { chorioamnionitis (\%) }\end{array}$ & $46.6(650 / 1,396)$ & $59.4(38 / 64)$ & $0.045^{* *}$ \\
\hline Total $(n=1,616)$ & $\begin{array}{l}\text { Negative for ESBL-E } \\
(n=1,550)\end{array}$ & $\begin{array}{l}\text { Positive for ESBL-E } \\
(n=66)\end{array}$ & $P$-value \\
\hline Sex (male) (\%) & $56.2(871 / 1,550)$ & $47.0(31 / 66)$ & $0.180 * * *$ \\
\hline Birth weight (kg) & $1.64(0.95-2.15)$ & $1.07(0.76-1.73)$ & $<0.001^{*}$ \\
\hline $\begin{array}{l}\text { Small for gestational } \\
\text { age }(\%)\end{array}$ & $6.7(105 / 1,550)$ & $12.1(8 / 66)$ & $0.094^{* * *}$ \\
\hline $\begin{array}{l}\text { 1-min Apgar score of } \\
<4(\%)\end{array}$ & $9.4(145 / 1,550)$ & $9.1(6 / 66)$ & $0.943^{* * *}$ \\
\hline $\begin{array}{l}\text { 5-min Apgar score of } \\
<7(\%)\end{array}$ & $11.2(174 / 1,550)$ & $12.1(8 / 66)$ & $0.821^{* * *}$ \\
\hline Neonatal mortality (\%) & $5.9(92 / 1,550)$ & $10.6(7 / 66)$ & $0.207^{* * *}$ \\
\hline $\begin{array}{l}\text { Neonatal intensive care } \\
\text { unit admission rate }(\%)\end{array}$ & $86.6(1,343 / 1,550)$ & $93.9(62 / 66)$ & $0.098^{* * *}$ \\
\hline EONS (\%) & $2.5(39 / 1,550)$ & $7.6(5 / 66)$ & $0.040 * * *$ \\
\hline Proven EONS $(\%)^{+}$ & $1.1(17 / 1,550)$ & $6.0(4 / 66)$ & $0.002^{* * *}$ \\
\hline Suspected EONS (\%) & $1.4(22 / 1,550)$ & $1.5(1 / 66)$ & $0.948^{* * *}$ \\
\hline $\begin{array}{l}\text { Late-onset neonatal } \\
\text { sepsis }(\%)\end{array}$ & $4.1(64 / 1,550)$ & $6.1(4 / 66)$ & $0.521^{* * *}$ \\
\hline
\end{tabular}

*Wilcoxon rank-sum test, ${ }^{* *}$ Pearson's chi-square test, ${ }^{* * *}$ Generalized estimating equation

Data are presented as nos. (percentages) and medians (interquartile ranges).

${ }^{+}$Among neonates with proven EONS, sepsis by ESBL-E was not identified in the ESBL-E-negative patients but was identified in three ESBL-E-positive patients.

ESBL-E, extended-spectrum $\beta$-lactamase-producingEnterobacteriaceae ; EONS, early-onset neonatal sepsis. 